\title{
The fate of organic fermentation products in carbonate sediments
}

T.F. HUTCHINSON ${ }^{1}$, P.M. LEUNG ${ }^{2}$, D.L. CALlAHAN ${ }^{3}$, A.J. KESSLER ${ }^{4}$, W.W. WONG ${ }^{1}$, C. GREENING ${ }^{2}$, P.L.M. COOK ${ }^{1 *}$

${ }^{1}$ Water Studies Centre, School of Chemistry, Monash University, Clayton, VIC 3800, Australia (*correspondence: perran.cook@monash.edu) ${ }^{2}$ School of Biomedical Sciences, Monash University, Clayton, VIC 3800, Australia

${ }^{3}$ School of Life \& Environmental Sciences, Deakin University, Burwood, VIC 3125, Australia

${ }^{4}$ School of Earth, Atmosphere \& Environment, Monash University, Clayton, VIC 3800, Australia

Permeable marine sediments are dynamic environments, with oxic-anoxic boundaries constantly changing due to wave oscillations and tidal cycles. As such, this environment selects for metabolically flexible bacteria, with fermentation dominating and remaining uncoupled from terminal respiration processes [1-2]. Despite these recent findings, we lack a complete understanding of the fermentation pathway and the question remains as to the fate of the metabolic endproducts. To date, this research has primarily been undertaken in temperate silicate sediments, while here we present work from metabolically active carbonate sediments of Heron Island, Australia. Using biogeochemical analyses and metabolomics we will present a comprehensive mass balance of carbon mineralization and electron acceptors utilized. In combination with metagenomics, we will further explore the possibility that bacteria are storing energy intracellularly via molecules such as glycogen, polyhydroxyalkanoates (PHAs), triacylglycerols (TAGs) and wax esters (WEs).

[1] Bourke et al. (2017) Nat. Geosci. 10, 30-35.

[2] Kessler et al. (2019) Nat. Microbiol. 4 1014-1023. 